\title{
Gradhiva
}

GRADHIV

Revue d'anthropologie et d'histoire des arts

$20 \mid 2014$

Création fiction

\section{D'aujourd'hui à avant-hier. Un évolutionnisme bien tempéré}

Gérard Lenclud

\section{OpenEdition}

1 Journals

Édition électronique

URL : http://journals.openedition.org/gradhiva/2907

DOI : 10.4000/gradhiva.2907

ISSN : 1760-849X

Éditeur

Musée du quai Branly Jacques Chirac

Édition imprimée

Date de publication : 1 octobre 2014

Pagination : 268-279

ISBN : 978-2-35744-074-6

ISSN : 0764-8928

Référence électronique

Gérard Lenclud, «D'aujourd'hui à avant-hier. Un évolutionnisme bien tempéré », Gradhiva [En ligne], 20 |

2014, mis en ligne le 01 octobre 2017, consulté le 22 septembre 2020. URL : http://

journals.openedition.org/gradhiva/2907 ; DOI : https://doi.org/10.4000/gradhiva.2907

(c) musée du quai Branly 


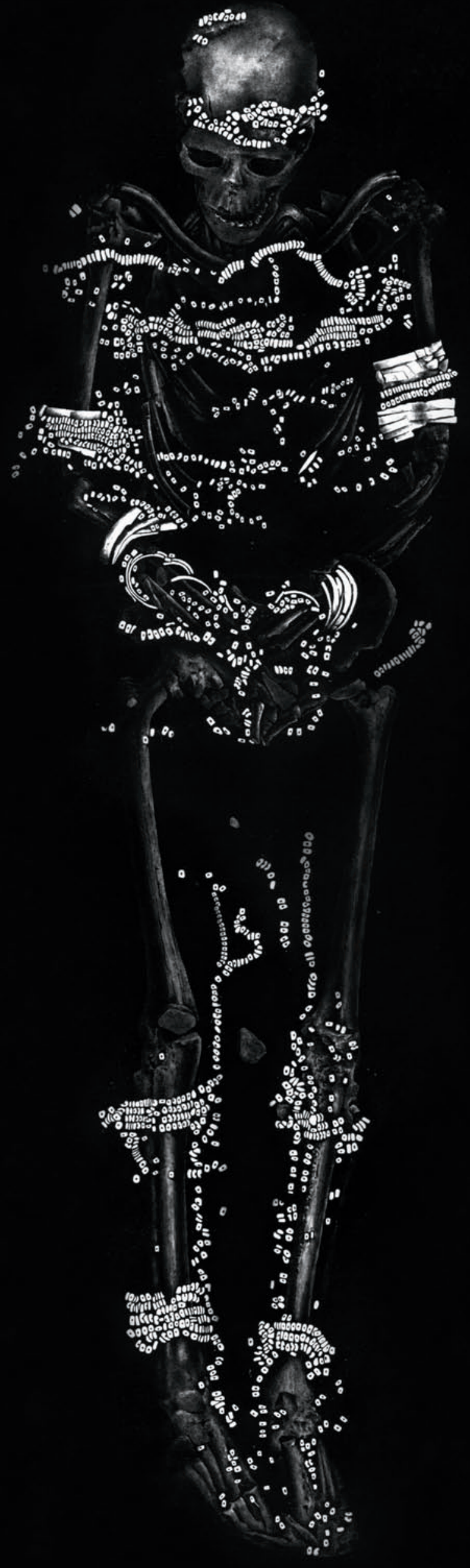




\section{D'aujourd'hui à avant-hier. Un évolutionnisme bien tempéré.}

par Gérard Lenclud

Alain Testart, Avant l'histoire. L'évolution des sociétés

de Lascaux à Carnac. Paris, Gallimard, 2012

L'ouvrage d'Alain Testart, Avant l'histoire. L'évolution des sociétés de Lascaux à Carnac répond à une ambition qui n'est pas mince: reconstituer dans leurs grandes lignes les formes de la vie sociale au Paléolithique supérieur, entre 40000 et 10000 av. J.-C., et dégager par là même les facteurs de l'évolution des sociétés à l'œuvre, comme l'indique le sous-titre du livre, entre Lascaux et ses peintures murales, datant d'environ 15000 ans av. J.-C., et Carnac avec ses tumulus, érigés dans la seconde moitié du ve millénaire avant notre ère.

Singulier projet de la part d'un anthropologue, diront peut-être à l'avance certains, attachés à la séparation des domaines scientifiques et ignorant l'itinéraire intellectuel de Testart. Ce serait à plus d'un titre faire erreur. D'abord il va sans dire, mais mieux en le disant, que les travaux des préhistoriens ont une répercussion anthropologique; il serait tout de même curieux que les hommes du Paléolithique supérieur, nos lointains semblables, soient exclus du champ de l'histoire humaine, et donc de la comparaison anthropologique, sous le prétexte de leur date de naissance ou du mode obligé d'en connaître. Rappelons ensuite, justement, que l'archéologie préhistorique emprunte nombre de ses concepts et de ses raisonnements à l'anthropologie. II se trouve qu'ici, et ce n'est pas la première fois, un anthropologue dûment familiarisé avec les problématiques, les méthodes et les caractères propres des données archéologiques intervient de son propre chef dans le champ de la préhistoire.

Si les préhistoriens font appel aux anthropologues, du moins en abordant certains sujets, et si Testart a plus d'une fois devancé l'appel, c'est en raison d'un fait assez évident. Assurément, la vie matérielle des chasseurscueilleurs du Paléolithique supérieur est de mieux en mieux connue grâce à l'accumulation de données archéologiques et aux avancées réalisées dans l'analyse de ces données. Force est de reconnaître, en revanche, que l'on ignore presque tout du type de société dans lequel ils vivaient. En effet, il est assez vain d'espérer extraire du sol des modes d'organisation sociale. Les structures de parenté, les principes d'échange, la présence de l'esclavage ou encore les formes d'exercice du pouvoir ne laissent guère de traces en terre. D'où la nécessité d'éclairer la signification des rares vestiges archéologiques portant témoignage, le plus souvent indirect, sur la vie sociale en les rapportant à la documentation ethnographique et aux modèles élaborés par l'anthropologie, bref de mettre en œuvre un comparatisme raisonné. C'est à quoi s'attelle Testart dans Avant l'histoire. 
1. Alain Testart a raison de considérer que l'idée, simpliste, selon laquelle les sociétés sans écriture ou sans État seraient "simples" fut un obstacle épistémologique majeur. La raison en est, me semblet-il, qu'il était difficile, et qu'il le reste, de se débarrasser tout à fait de l'impression malvenue qu'une société de chasseurs-cueilleurs, ou d'agriculteurs sur brûlis, est tout de même plus "rudimentaire», ou moins "évoluée ", qu'une société postindustrielle. Et si la pensée sociologique peine à résister à la tentation évolutionniste, c'est que la sociologie s'est édifiée dans certaines sociétés seulement; et cela, elle ne parvient pas à tout à fait l'oublier...

\section{double page} précédente

fig. 1

Dessin du squelette masculin Sunghir I avec ses parures de dents et d'ivoire, paléolithique supérieur (environ 25000 ans avant J.-C) , in Otto N. Bader, "Sungir : inhumations paléolitiques » article écrit en russe publié dans Nikolai O. Bader (dir.) Posdnepaleolitischeskoje posselenije Sungir, Moscou, Nautschny Mir 1998 : 5-160, planche 1. DR.
Présentons, pour commencer, certains des attendus sur lesquels se fonde l'ouvrage; ce sera au prix d'un schématisme inévitable.

Première question appelant réponse: Testart place son entreprise sous pavillon de l'évolutionnisme social; ne serait-il pas en retard d'un siècle? Son point de vue à l'égard des représentants de l'évolutionnisme social et culturel tranche, en effet, avec une opinion majoritaire en anthropologie. Pour dire vite, depuis Franz Boas, on a pris l'habitude dans la discipline d'en tresser l'éloge en dépit de la perspective évolutionniste adoptée (et à laquelle ils auraient eu bien du mal à ne pas adhérer tant elle imprégnait l'air du temps). On sait qu'ils entendaient élaborer une histoire naturelle de l'humanité sociale et culturelle. Or ils ont emprunté pour la rédiger un raccourci commode permettant de faire l'économie d'une «histoire détaillée», selon l'expression d'Edward Tylor, qu'ils n'auraient pas eu, au demeurant, les moyens de coucher par écrit. En effet, le modèle d'intelligibilité proposé consiste à rapporter l'ordination des phénomènes dans le temps à une ordination logique invariante, en introduisant la loi de développement selon laquelle le simple serait toujours antérieur au complexe. Ce qui fut devait être; ce qui arriva était écrit! C'est à bon compte, en quelque sorte, que le jeu du hasard était conjuré et la diversité humaine organisée. Or Testart, assez seul dans son cas, célèbre les mérites des représentants de l'évolutionnisme social aussi à cause de cette position évolutionniste. Il les félicite, en effet, de s'être mis en quête d'un ordre évolutif à partir de données contemporaines, même s'ils échouèrent pour des raisons qui, à ses yeux, ne tiennent pas toutes à eux.

Résumons drastiquement. À leur crédit, le projet de l'entreprise à mettre en œuvre et, à cette occasion, la découverte capitale du fait qu'une société laisse entrevoir des niveaux distincts d'organisation, entretenant entre eux des relations de correspondance ou se révélant en décalage les uns par rapport aux autres, susceptibles d'évoluer à des rythmes différents. À leur débit, en particulier, l'idée funeste que des sociétés seraient plus simples que d'autres ou encore l'hypothèse du "parallélisme » selon laquelle toutes les sociétés évolueraient conformément à une même loi de trajectoire, parcourant les mêmes étapes, franchissant les mêmes seuils, jusqu'à se faire (provisoirement) victoriennes. Une excuse, si l'on peut dire: les représentants de l'évolutionnisme anthropologique ne disposaient pas des moyens de rédiger l'histoire, même peu «détaillée», de l'humanité sociale. Il leur manquait, et pour cause, tant les données de l'archéologie que celles de l'ethnographie.

Que se passe-t-il si, à l'image de Testart, on ampute l'évolutionnisme social «réel », au sens où l'on parle de communisme réel, à la fois du modèle logique de déroulement où le simple (l'inférieur) est au départ et le complexe (le supérieur) à l'arrivée ${ }^{1}$ et des schémas de développement comportant des stades nécessairement atteints puis dépassés par toutes les sociétés? On tend à commuer une doctrine, voire une idéologie, tissée par les évidences axiologiques d'une époque révolue, en programme de recherche. Certes, ce dernier repose sur des propositions théoriques mais elles sont modestes, du moins de prime abord. L'idée que le hasard seul présiderait à la transformation des sociétés et que tous les ordres de succession seraient 
concevables ou même advenus heurte pour le moins le sens commun sociologique, et même historique. (Mais de là à évoquer des lois de transformation ou de succession, c'est une autre affaire!)

Le refus de cette idée constitue un bon morceau du parti pris théorique de Testart, assez proche, par conséquent, de ce que je suis tenté d'appeler un évolutionnisme méthodologique. II épouse certes le programme évolutionniste $d u x x^{e}$ siècle mais sans adopter aucune des conclusions anthropologiques qu'en tirèrent ses artisans. Et ce programme évolutionniste, il en baptise généreusement le vin: «L'évolutionnisme, c'est le changement, l'histoire, plus la notion d'ordre [...]. Penser une évolution, c'est tenter de faire coïncider un ordre qui est temporel avec un autre qui ne l'est pas, du moins avec un ordre qu'il est possible de situer dans le temps, mais qui n'est pas défini en fonction de lui » (p. 82).

Deuxième point méritant l'attention: l'acception conférée par Testart à la notion de société. Une société est pour lui un «ensemble de structures et d'institutions». Elle ne consiste aucunement dans les hommes (le groupe, la communauté, le peuple, la nation...) dont on dirait, à tort donc, qu'elle est composée. Le terme de société est sous sa plume synonyme de l'expression "modèle de société». II désigne une formule de coexistence entre les hommes, parfaitement «indépendante», écrit-il, de ces derniers. Et, à cette occasion, il durcit l'opposition consacrée entre société et culture, par conséquent entre anthropologie sociale et culturelle. En effet, et ce serait selon Testart le propre de l'approche sociologique, on pourrait étudier une société en faisant abstraction du peuple ayant adopté ses institutions; le tenant de l'anthropologie sociale ferait en somme œuvre de constitutionnaliste. II serait impossible, en revanche, de se pencher sur une culture sans prendre en compte les hommes qui la font exister. À la différence d'une société, une culture serait unique en son genre. Plusieurs sociétés pourraient partager le même état social tout en se distinguant les unes des autres par leurs cultures, même si, doit bien admettre Testart, «les institutions contribuent à faire la culture». II s'ensuit que «la conception d'une évolution des formes sociales dans la préhistoire ne se résume pas à un tableau des cultures archéologiques dans leur succession chronologique» (p. 94).

Que l'histoire des sociétés, celle qui se raconte, ne se confonde pas avec leur évolution, qui est à décrypter, c'est une affaire entendue aux yeux de l'auteur d'Avant l'histoire. Mais qui dit évolution (des modèles de société), ou plus exactement évolutions au pluriel, car elles peuvent être divergentes au contraire de ce qui se passe en histoire naturelle, semble vouloir dire mécanisme, responsable des enchaînements. Or en abordant la question du pourquoi de l'évolution sociale, Testart rejette tour à tour le primat explicatif conféré en dernière instance au développement des techniques, à l'environnement, à la démographie ou à la religion. La diffusion ne saurait être davantage promue, selon lui, en processus unique, ni même déterminant, de l'évolution. (Mais pourquoi, entre parenthèses, faudrait-il se lancer à la poursuite d'une causalité unique?) La conclusion tirée de ces refus en chaîne est qu'en ce qui concerne les sociétés humaines, il n'est pas nécessaire de concevoir le jeu d'un mécanisme. Pourquoi cela? Citons un peu longuement Testart: «Parce que l'action des hommes suffit à transformer leurs sociétés. 
2. La rétrodiction est au menu quotidien de l'historien. Un événement étant déjà survenu, une situation déjà produite, la rétrodiction consiste à évaluer la probabilité respective des hypothèses explicatives en présence.
Peut-être ne les transforment-ils pas toujours conformément aux fins qu'ils s'étaient proposées, peut-être leur action produit-elle régulièrement des résultats non escomptés et parfois contraires à leurs souhaits, mais c'est toujours par le biais de leurs actions que se réalisent ces transformations. L'action humaine (de l'homme en société, agissant sur la société) est une condition nécessaire et suffisante de l'évolution des sociétés" (p. 149). Belle page d'anthropologie (philosophique) qui mériterait d'ailleurs discussion. Elle suggère en tout cas l'idée d'une causalité circulaire. En effet, les motivations des acteurs, ingrédient obligé car définitoire de toute action humaine, trouvent évidemment une bonne part de leur logique dans les institutions sociales, ces institutions sur lesquelles elles exercent un pouvoir causatif. Et si tel est le cas, c'est parce que les institutions sociales cristallisent des idées et des valeurs, renferment des sanctions positives et négatives, les unes et les autres au fondement des raisons d'agir. Bref, c'est à l'intérieur des institutions sociales qu'il faudrait chercher le moteur de leur transformation et de leur succession.

Quelques mots enfin sur le recours aux données ethnographiques (ou historiques) afin de remédier à la quasi-invisibilité des institutions sociales en archéologie. À coup sûr, nul ne songe plus à postuler une sorte d'équivalence en matière d'état social entre «les Germains de Tacite et les PeauxRouges américains" à la façon de Friedrich Engels. Personne n'imagine a fortiori que des communautés humaines aient pu, des millénaires durant, persévérer dans leur être social, structurées à l'identique. Aucun préhistorien n'a dans l'idée, faisant retour à «la vieille méthode illustrative par le parallèle ethnographique", d'affubler l'homme préhistorique de défroques empruntées aux Fuégiens ou aux Australiens. II s'agit de chercher dans la documentation ethnographique de quoi tenter de répondre à certaines des questions que se pose l'archéologue, désarmé face aux lacunes de sa propre documentation. Le comparatisme est raisonné; il s'impose des contraintes. II en est de l'archéologue dans ce cas, et de Testart dans cet ouvrage, comme de l'historien faisant de l'histoire comparée (ou de l'anthropologue mettant en perspective des descriptions ethnographiques): la comparaison est une heuristique. «Et si, par hasard, c'est de ce côté qu'il fallait creuser?» Elle permet d'élargir le spectre des hypothèses explicatives. L'opération débouchet-elle sur la découverte de relations nécessaires ou d'incompatibilité absolue entre plusieurs traits? Testart en est pour sa part convaincu.

Que peuvent donc bien nous suggérer les sociétés de chasseurscueilleurs actuels, mais surtout subactuels, en ce qui concerne les institutions sociales au Paléolithique supérieur? Je vais me contenter de présenter deux ou trois étapes décisives dans l'argumentation touffue développée par Testart dans la seconde partie d'Avant l'histoire. Ces raisonnements sont nourris par l'érudition ethnographique de l'auteur; cela n'empêche évidemment pas qu'ils soient soumis aux risques inhérents à l'extrapolation et aux aléas de la rétrodiction ${ }^{2}$.

Tout d'abord, vers quelles sociétés de chasseurs-cueilleurs du présent, et plus encore du passé proche, se tourner? Comme le rappelle à juste titre Testart, il existe bien des façons de s'organiser en société chez ceux de nos contemporains, ou presque contemporains, qui n'ont pas emprunté le 
tournant du Néolithique. Le stockage, en particulier, ferait la différence entre eux tous. La conservation à grande échelle de ressources alimentaires non domestiquées, saisonnières mais abondantes, entraine des conséquences sociales importantes. Elle permet la sédentarité; elle autorise une densité démographique élevée; elle commue des biens matériels en éléments de richesse; elle est source d'inégalités économiques liées à la richesse. C'est ainsi que, à égalité chasseurs-cueilleurs en ce sens que ni les uns ni les autres ne font de part à l'agriculture ou à l'élevage, un fossé sépare les «riches» sédentaires stockeurs de la côte nord-ouest de l'Amérique du Nord et les «pauvres» nomades d'Afrique, Pygmées ou San, ou d'Australie. Kwakiutl, Haïda ou Tlingit ont édifié la civilisation flamboyante que l'on sait, déployée par une société esclavagiste, fortement stratifiée, structurée par la richesse et marquée par des comportements ostentatoires. Rien de tel dans la forêt équatoriale africaine, dans le Kalahari ou l'hinterland australien. Ces sociétés-là de chasseurs-cueilleurs ont formé pour leur part ce que Testart nomme des sociétés «achrématiques». Les biens matériels y sont évidemment présents mais ils ne constituent pas une richesse socialement utile, donnant accès à la réputation et au pouvoir, alimentant des institutions tels le prix de la fiancée ou le wergeld. Si ces sociétés ne sont pas égalitaires, elles ignorent les inégalités économiques.

Ce sont ces sociétés qui ont le plus souvent servi de modèle, ou de référence, aux préhistoriens pour esquisser à grands traits le portrait sociologique vraisemblable des chasseurs-cueilleurs du Paléolithique supérieur. À bon droit, selon Testart. En effet, il réfute la thèse soutenue par l'archéologue Brian Hayden, sur la base des sépultures découvertes à Sungir, à l'est de Moscou, pour qui il est parfaitement concevable que, dans certaines régions d'Europe, se soient construites au Magdalénien des sociétés comparables à celles de la côte Nord-Ouest. Testart rejette cette suggestion et la possibilité que les conditions sociales aient pu considérablement varier au sein de populations occupant des écosystèmes contrastés. Aucune donnée archéologique ne militerait en faveur de la sédentarité, ici ou là; aucune n'indiquerait vraiment la présence d'une richesse fondant des inégalités de destin personnel ou collectif; aucune ne livrerait les traces de pratiques ostentatoires élevées à la hauteur d'un art de faire social.

Pour être toutes "achrématiques", ces sociétés de chasseurscueilleurs nomades du Paléolithique supérieur ne sauraient être dites, par décret de méthode, avoir partagé les mêmes institutions sociales. La documentation ethnographique montre, en tout cas, que deux modèles de société très différents sont compatibles avec le régime de chasse-cueillette itinérante. L'opposition entre ces modèles, déjà explicitée par Testart dans ses Éléments de classification des sociétés (2005), mobilise trois traits: la présence ou l'absence d'un système clos de classification des choses, des animaux et des hommes, la manière de prendre époux et de s'acquitter des prestations liées aux épousailles, la logique prévalant dans le partage du gibier. Les sociétés de chasseurs-cueilleurs nomades dites de type A, exemplifiées par les Aborigènes d'Australie (sinon représentées par eux seuls), développant totémisme et exogamie, insèrent donc les humains dans un cadre classificatoire intangible et à extension universelle; le mariage s'y accompagne d'obligations à vie sous la forme de services; le chasseur 
ci-contre

fig. 2

Vue de la partie supérieure du squelette Sunghir I, in Otto N. Bader, "Sungir : inhumations paléolitiques * article écrit en russe publié dans Nikolai O. Bader (dir.)

Posdnepaleolitischeskoje posselenije Sungir, Moscou, Nautschny Mir 1998 : 5-160, planche 2. DR. n'est pas propriétaire de son butin et n'est donc pas l'opérateur social de sa distribution. Les sociétés de chasseurs-cueilleurs nomades de type B ignorent tout principe systématique de classification déterminant la position de chacun; on y pratique le service pour la fiancée qui, une fois effectué, libère le gendre de toute obligation; la chasse donne lieu à de véritables systèmes juridiques de répartition de son produit mais celui, ou ceux, qui a contribué à la prise de l'animal est reconnu comme l'ayant droit principal et, à ce titre, comme le «fondé» de redistribution. À bien suivre Testart, l'existence, ou non, d'un système classificatoire enserrant les êtres humains dans les mailles de son filet déterminerait la forme des prestations liées au mariage et les modalités de partage du gibier.

Auquel de ces deux modèles d'état social rapporter les sociétés paléolithiques? La réponse à la question se trouverait, avance Testart, dans les caractéristiques de l'art pariétal. II observe qu'à la différence des animaux, représentés dans les fresques et les gravures avec un réalisme appuyé mais s'arrêtant aux frontières de l'espèce, les humains apparaissent sous les traits de créatures composites ou inachevées. Contrastant de ce point de vue avec l'art mobilier, l'art pariétal paléolithique livre l'image d'une humanité hybride, incomplètement dégagée du règne animal. Preuve est faite, pour Testart, de la prégnance au Paléolithique supérieur d'une vision totémique du monde: les animaux, classés selon leurs espèces, renverraient aux hommes, classés selon des divisions sociales. Mais, objectera-t-on, pourquoi ces humains qui ne le sont pas à part entière? Pourquoi sont-ils bestialisés sur les parois des grottes? Testart tient sa réponse prête: la pensée totémique «s'exprime typiquement par l'imagination d'un temps mythique pendant lequel les hommes étaient encore mal différenciés des animaux, alors même que ceux-là étaient déjà différenciés en espèces » (p. 267). Les représentations iconographiques de l'art pariétal, au plus profond des cavernes, seraient en somme un précurseur des mythologies de l'Australie. On l'a compris: les sociétés de chasseurs-cueilleurs du Paléolithique supérieur, toutes «achrématiques », auraient été selon Testart du type $A$, incarné par les Aborigènes d'Australie.

Cette opposition entre sociétés des types A et B est au cœur des schémas d'évolution proposés dans Avant l'histoire, conduisant par divers itinéraires des structures sociales paléolithiques à celles qui constitueront le moteur des innovations durant la courte période de l'Épipaléolithique, précédant et orientant le mode d'entrée dans le Néolithique. En effet, ces deux modèles de société présentent selon Testart un potentiel évolutif très contrasté. Les institutions caractérisant le type A ne comportent en ellesmêmes aucune incitation à développer et augmenter la production de biens matériels. Chaque individu trouve à sa naissance le destin qui sera sien, sa vie durant. Impossible de l'infléchir, encore moins de le transformer. Ce destin est inscrit dans la charte sociale du groupe à la manière dont, chez les Aborigènes d'Australie, le sort matrimonial des hommes et des femmes est déterminé par l'appartenance à des classes qui ont maints attributs de la caste, hormis évidemment l'exogamie et la hiérarchie. Dans les sociétés de type $\mathrm{A}$, la personne est prise dans un réseau d'obligations dont elle ne saurait se libérer. Dans ces conditions, à quoi bon produire plus ou mieux, au-delà du nécessaire? La détention de biens matériels non commués, 


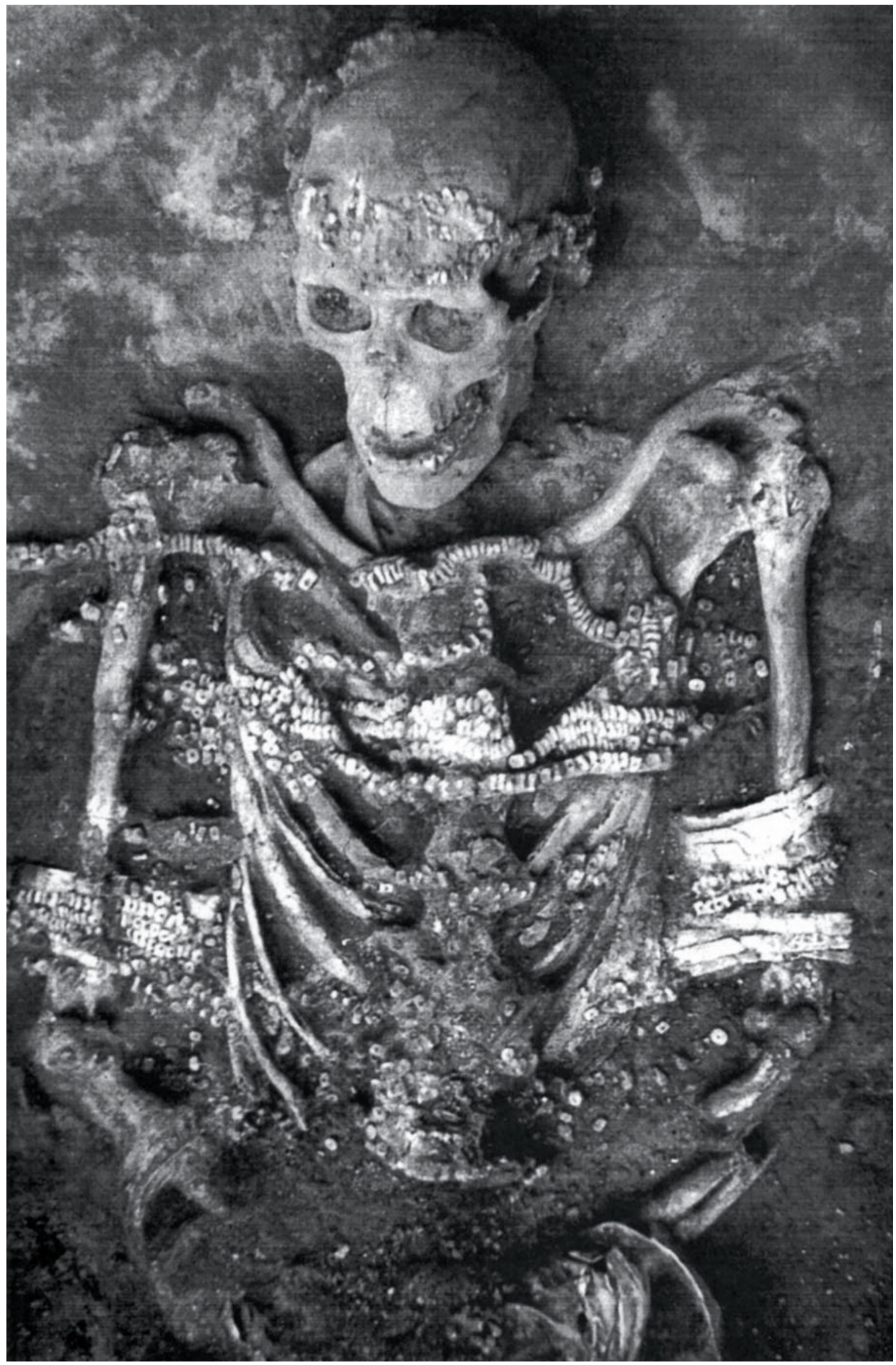


ni surtout non commuables, en richesse ne procure pas de considération sociale; ces biens ne peuvent davantage servir à s'affranchir de devoirs socialement prescrits. Pour reprendre une vieille distinction introduite par Henry Sumner Maine, les sociétés de type A seraient des sociétés «à statut ».

À l'opposé, les sociétés de type B seraient des sociétés «à contrat ». Loin d'être fixé à l'avance, imposé de l'extérieur, le destin personnel serait en partie négociable. II n'est pas déterminé, comme dans les sociétés de type A, par la définition sociale de celui qu'on est en arrivant au monde et qu'on continuera d'être mais par la prise en compte de ce que fait l'individu et qui, dans une certaine mesure, est consenti. Ce dernier ne dispose-t-il pas de la capacité à se libérer de ses obligations en payant de sa personne (en attendant de s'acquitter de ce prélèvement libératoire par le truchement de biens matériels durables)? Les institutions spécifiant ce modèle de société fabriqueraient un milieu social propice à l'expérimentation, donc aux inventions et aux innovations.

Que les institutions sociales du Paléolithique supérieur aient été du type $A$, donc guère susceptibles de pousser les hommes au progrès technique, Testart en veut la preuve notamment dans ce qu'il estime être le tout petit nombre des inventions majeures entre 40000 et 10000 av. J.-C., refusant d'élever à ce rang tant le débitage lamellaire que la miniaturisation de l'outillage lithique (déjà à l'œuvre d'ailleurs, à ma connaissance, en Afrique au Middle Stone Age). En revanche, on assiste à une accélération notable des inventions et des innovations durant les quelque cinq mille ans du Mésolithique (ou Épipaléolithique).

Vers 10000 av. J.-C., par conséquent, à l'aube de cette période mal taillée d'où naît le Néolithique, il se produit un changement de rythme considérable. C'est donc, dans la logique du raisonnement de Testart, que des sociétés relevant du type $A$ se sont converties à la constitution sociale régissant les sociétés dites de type $B$. Comment cela s'est-il produit? Peut-être ai-je mal lu Avant l'histoire mais je n'y ai pas trouvé de réponse franche et nette à la question, seulement des éléments d'explication dispersés dans plusieurs chapitres. Oublions cela et lançons un coup d'œil sur ce qui se passe, ou plutôt ne se passe pas, chez des chasseurs-cueilleurs qui sont restés de type A sans que rien ne les y oblige, en l'occurrence les Aborigènes d'Australie. Ils ne vont ni inventer ni adopter l'agriculture. Si l'on considère avec Testart qu'environ un quart du territoire australien s'y prête, en particulier l'Australie tropicale au genre d'horticulture pratiqué en Papouasie du Sud-Est, force est d'admettre l'absence de compatibilité entre les idées/valeurs au cœur des institutions sociales aborigènes et le mode de vie agricole, ou «semi-agricole», impliquant l'existence sédentaire dans des villages.

Voyons maintenant le chemin que vont parcourir, à vive allure pour certaines puis plus vive encore, les sociétés de chasseurs-cueilleurs de type B (mais anciennement de type A) dont les institutions stimulent la tendance à innover. II mène à l'invention de l'agriculture. «Ce sont les structures sociales de type $\mathrm{B}$ qui portent les hommes qui relèvent de ce type à inventer l'agriculture» (p. 349). N'allons pas exagérer, recommande Testart après 
d'autres, l'opposition entre le chasseur-cueilleur et l'agriculteur ou l'éleveur; l'existence de chasseurs-cueilleurs sédentaires stockeurs conduit à la relativiser et, peut-être même, à assigner au stockage certaines des propriétés et conséquences sociales généralement attribuées à l'agriculture. En effet, lorsque le stockage concerne la nourriture, il entraîne la transformation de produits alimentaires en biens meubles et durables, susceptibles d'être épargnés, investis, échangés ou encore prêtés. L'invention, ou l'adoption, de l'agriculture est moins celle, technique, de méthodes culturales, bref de façons de faire, que celle, sociologique, d'un mode de vie, allant de pair avec un changement dans les habitudes sociales et dans les représentations du monde, bref invention, ou adoption, de façons d'être.

D'où la nécessité, selon Testart, d'insérer la dimension technique de l'invention, ou de l'adoption, de l'agriculture dans ce mouvement d'expérimentation générale qui emporte les sociétés de type B. L'enchaînement causal serait alors celui qui suit. 1) L'accroissement des forces productives, dont l'aiguillon est social, est la cause du processus de sédentarisation, du moins là où le stockage était absent. Les équipements du chasseur s'alourdissent, malgré la miniaturisation, et entravent la mobilité; l'existence de réserves alimentaires supprime l'obligation du déplacement. 2) L'adoption de la sédentarité est à son tour la cause du passage au mode d'existence social de l'agriculteur, "cause, à court terme, de la petite agriculture et, à long terme, de l'adoption finale d'une économie agricole» (p. 351).

Et l'on trouve dans Avant l'histoire des schémas d'évolution, divergents, des sociétés de chasseurs-cueilleurs nomades du Paléolithique supérieur, fondés sur les différences de structures repérées dans la documentation ethnographique, intégrant aussi - le moyen de faire autrement - les contraintes environnementales.

Une autre invention sur laquelle Testart insiste tout au long de l'ouvrage est celle de la richesse, bien dans «le sens de l'histoire» (intitulé de l'un des chapitres du livre). Invention, avons-nous vu, corrélée déjà au stockage en régime de chasse-cueillette. Je me contenterai à ce sujet de signaler la manière dont Testart en imagine l'apparition. Elle semble contredire, notons-le au passage, ce qu'il affirme à propos de la création des institutions, et la richesse en est une. Testart pose comme principe que l'invention d'une technique n'a pas pour origine la volonté des hommes d'en faire tel ou tel usage. À l'opposé, une institution sociale serait construite dans un certain but. Le but peut n'être pas atteint mais, à supposer qu'il le soit, les conséquences de la mise sur pied de l'institution doivent être considérées comme intentionnelles. L'invention de la richesse aurait-elle répondu à certains objectifs? Non, répond Testart, elle serait arrivée toute seule, en quelque sorte, à l'insu de ses inventeurs ou sans que ces derniers y prennent garde. Toutefois ces biens matériels, qui sont à la richesse ce que son marbre est à la statue, doivent être institués en richesse. Cela arriva «le jour où le détenteur d'une obligation accepta de recevoir en lieu et place du travail auquel cette obligation lui donnait droit des produits matériels durables, que lui-même pourrait donc utiliser de la même façon » (p. 405). La richesse naquit donc avec le prix de la fiancée et le wergeld, attributs des sociétés de type B. Et, ici encore, Testart propose 


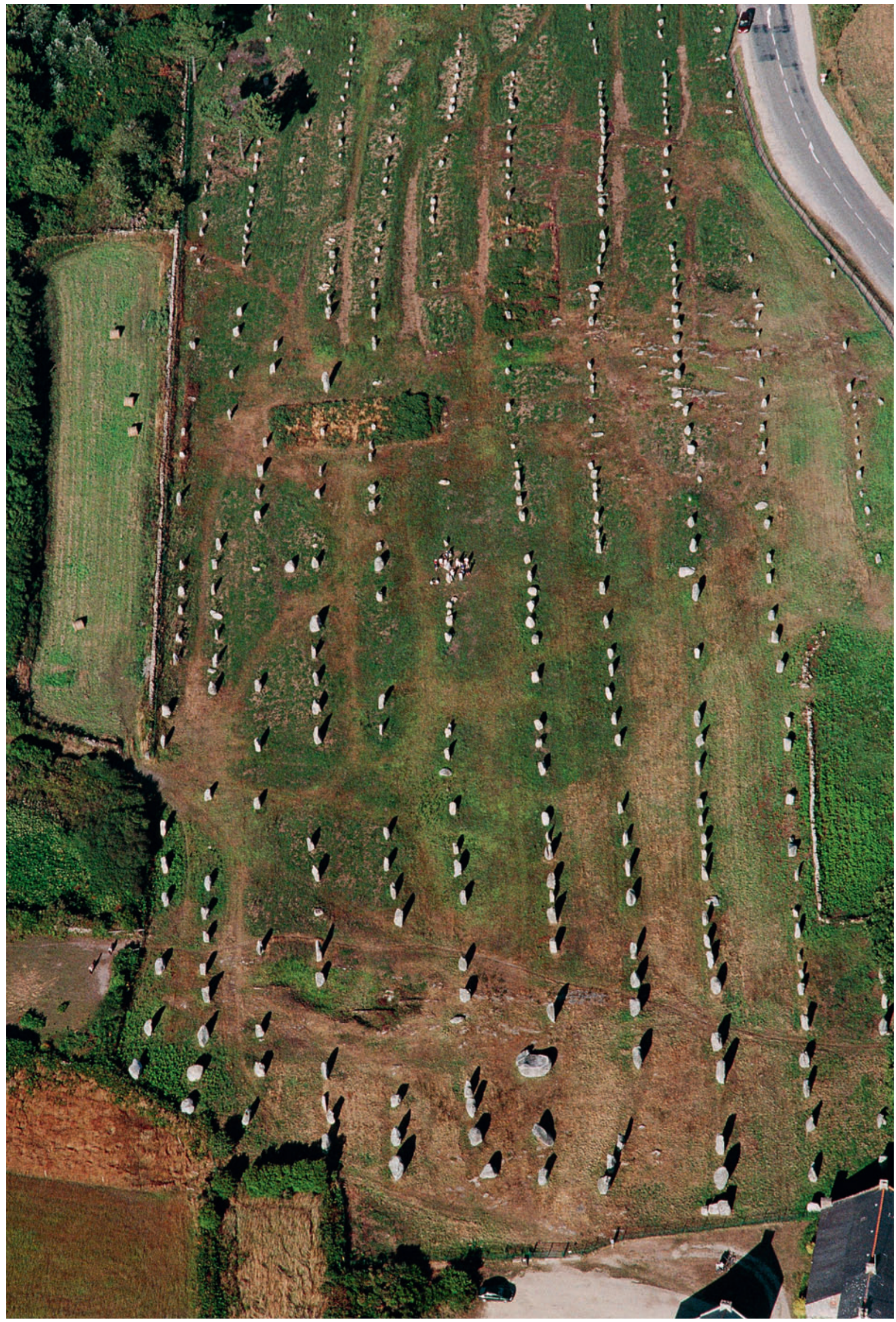


des scénarios d'évolution, du Paléolithique supérieur au Néolithique, fondés à la fois sur la présence ou l'absence de la richesse et sur les deux formes qu'il retient de la propriété foncière.

J'arrêterai ici la recension de cet ouvrage, laissant de côté en particulier les développements abondants consacrés à l'évolution des systèmes politiques de la préhistoire. Les pages qui précèdent suffiront, du moins je l'espère, à donner au lecteur une idée de la méthode mise en œuvre par Testart et du genre d'argumentation qu'il développe.

II revient évidemment aux spécialistes, les archéologues, parfois rudement pris à partie par l'auteur, de discuter les thèses soutenues dans le livre et d'évaluer son apport à la connaissance des sociétés préhistoriques. Ils admettront, me semble-t-il, l'idée constamment mise en avant par Testart selon laquelle les raisonnements archéologiques ne peuvent s'appuyer seulement sur les données de l'archéologie. Ils rappelleront que seules, en revanche, ces dernières sont à même de valider un raisonnement archéologique. Aux archéologues donc de dire si celles mobilisées par Testart le sont à bon escient et étayent ses propres raisonnements.

J'ai envie pour terminer de revenir d'un mot sur ce que j'ai écrit plus haut, à savoir que les attendus théoriques justifiant l'écriture d'Avant I'histoire, et l'évolutionnisme bien tempéré qui s'y exprime, ne sont modestes que de prime abord. À coup sûr, il se trouvera des lecteurs qui, tout en saluant l'érudition de l'auteur et en s'inclinant devant l'ingéniosité de certaines de ses analyses, tiqueront sur le fond de sa démarche. Ce sont ceux qui, revêtus des habits historicistes ou culturalistes dont Testart dénonce ici ou là le port, combattent le réalisme conceptuel dans les sciences (historiques) de l'homme. Ils s'y emploient au nom d'un certain nominalisme historique. Ils professent que les espèces, ou les types, ne se laissent véritablement distinguer des individus qu'en histoire naturelle. Le projet d'Avant l'histoire relève du pari exactement contraire ${ }^{3}$.
3. À peine avais-je entamé la lecture d'Avant l'histoire que j'appris la disparition de son auteur. Ainsi donc cet ouvrage sera-t-il le dernier publié du vivant d'Alain Testart (mais pas son ultime œuvre puisqu'un nouveau livre, Art et religion au Paléolithique supérieur. est annoncé comme à paraître dans le chapitre consacré à l'art pariétal).

Il m'a été impossible d'en faire abstraction en rendant compte de cet ouvrage tant celui-ci porte sa marque, celle d'un chercheur de conviction. En lisant certaines pages, je croyais l'entendre écarter les objections, celles qu'entre autres je lui aurais amicalement adressées de vive voix et qu'il aurait non moins amicalement repoussées, de façon fort catégorique. Cela s'était passé ainsi lors de la parution de son Essai d'épistémologie en 1991. Combien j'aurais aimé que cela puisse se produire à nouveau, vingt ans plus tard.

\section{ci-contre}

fig. 3

Alignements mégalithiques du Menec, Carnac, néolithique moyen ou final (4000 à 2000 ans avant J.-C.).

Photo Georg Gerster/Rapho. 